\title{
AVALIAÇÃO DO GERENCIAMENTO DE RISCOS PARA DESENVOLVIMENTO DE NOVOS PRODUTOS E TECNOLOGIA: REVISÃO INTEGRATIVA DE LITERATURA
}

\author{
Sara Marques O. A. Souza ${ }^{1}$; Valter Estevão Beal ${ }^{2}$; \\ ${ }^{1}$ SENAI CIMATEC, Avenida Orlando Gomes, Salvador Bahia; sm_oa1@hotmail.com; \\ ${ }^{2}$ SENAI CIMATEC, Avenida Orlando Gomes, Salvador Bahia
}

Resumo: As ferramentas ou modelos de gerenciamento de risco têm sido propostos com objetivo de controlar e tratar o risco em projetos industriais. Porém, o que se percebe é um modelo padrão aplicados a diferentes tipos de projetos, inclusive projetos de desenvolvimento de novos produtos e tecnologias. Como gerenciar riscos no desenvolvimento de produtos e tecnologias, já que para tal, é necessário considerar o nível de maturidade do processo? Este estudo realiza uma revisão integrativa da literatura, sobre a gestão de riscos no desenvolvimento de produtos e tecnologia, considerando o uso da métrica TRL (Technology Readiness Level), abordando também riscos referentes ao Meio Ambiente e Segurança. Dos 307 artigos encontrados, apenas 10 corresponderam às expectativas, ao que concerne atendimento às questões norteadoras da pesquisa.

Palavras-Chave: Risco; TRL; Tecnologia; Produto; Desenvolvimento.

\section{RISK MANAGEMENT ASSESSMENT FOR NEW PRODUCTS DEVELOPMENT AND TECHNOLOGY: INTEGRATIVE LITERATURE REVIEW}

\begin{abstract}
Risk management tools or models have been proposed to control and address risk in industrial projects. However, what is perceived is a standard model applied to different types of projects, including projects for the development of new products and technologies. How to manage risk in product and technology development projects, as it is necessary to consider the level of technology maturity? This study performs an integrative assessment of the literature on risk management in product and technology development considering the TRL (Technology Readiness Level) and considering also Environment and Safety risks. Search results were analyzed and identified. Of the 307 articles found, only 10 corresponded to expectations, which concerns meeting the guiding questions of the research.
\end{abstract}

Keywords: Risk; TRL; Technology; Product; Development 


\section{INTRODUÇÃO}

Devido à complexidade do desenvolvimento de tecnologias, tal como a variedade de informações que estes projetos estão submetidos, uma quantidade considerável de fatores de riscos necessita de constante atenção. Além disso, o projeto de desenvolvimento é frequentemente exposto a riscos (ou ameaças), relacionados as suas atividades, métodos, equipe e ao processo envolvido em cada fase do projeto [1]. Outro fator que deve ser avaliado, são as métricas de gestão dessas ameaças e como as mesmas podem agregar valor no processo. A validação dessas métricas deve considerar as peculiaridades de cada projeto, verificando se apenas um único método é suficiente para ser aplicado a todos tipos de projetos [2].

Diante disso, este estudo realiza uma revisão integrativa da literatura sobre a gestão de riscos no desenvolvimento de produtos e tecnologia considerando a métrica TRL (Technology Readiness Level), abordando também riscos referentes ao Meio Ambiente e Segurança. Todos os possíveis eventos desfavoráveis (técnicos, gerenciais, ambientais e de segurança), devem ser gerenciados para reduzir sua probabilidade de ocorrência em cada fase do desenvolvimento.

\subsection{Gestão de Projeto}

Usar métodos ou formas para conduzir projetos, é importante para lidar com eventos novos e complexos, considerando as dinâmicas ambientais [3]. A multidisciplinaridade, é um dos fatores que contribuem para a exposição de riscos em um projeto [4]. Quanto mais complexo for o projeto, maior integração será necessária para comunicação e resolução dos riscos.

\subsection{Gestão de Riscos}

O gerenciamento de riscos avalia todas as atividades em uma organização. Isso inclui envolvimento constante com as partes interessadas, considerando o contexto ambiental, da gestão, do desenvolvimento do produto ou processo, tal como fatores humanos e culturais [5]. O que determina uma tarefa fundamental para a organização [6], mas muitas vezes não agregam valor ao processo, representando apenas como gerenciar um modo de falha de forma "automática". Usar análise de multi-categorias é uma importante prática, pois agrega diferentes fatores. Essa análise possui elevado valor agregado, pelo seu escopo altamente complexo, já que envolve sistemas e subsistemas, equipes multidisciplinares, incertezas tecnológicas e necessidade de atendimento a leis nacionais e internacionais [7]. A análise da probabilidade e da consequência do risco são fatores que devem ser priorizados em uma análise de risco [8]. Como consequência, essa análise deve informar o nível de impacto que foi considerada sobre o pior cenário. Assim, o cruzamento entre a severidade ou impacto do risco e a probabilidade de ocorrência, resulta na criticidade do risco. Logo, deve-se considerar riscos inerentes ao processo (conservação de energia, materiais, emissão de resíduos tóxicos e seus respectivos impactos ambientais) [9]. 


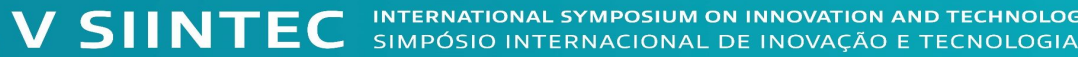

\begin{tabular}{l|l} 
CIRCULAR ECONOMY & ECONOMIA CIRCULAR
\end{tabular}

\subsection{Technology Readiness Level}

Por tratar-se de desenvolvimento de tecnologias e inovação, recomenda-se que o processo seja submetido a um método de qualificação das tecnologias agregadas. Isso garante a confiabilidade do sistema, através da integridade das informações envolvidas e a respectiva validação. Integrado a qualificação da tecnologia, ferramentas para avaliação da maturidade tecnológica de cada etapa do desenvolvimento (Technology Readiness Level ou TRL), deve ser considerado, para garantir o quão confiável é processo [10]. Por fim, uma metodologia para classificação de riscos, agrega valor no processo de desenvolvimento [11]. A seguir, segue os princípios da TRL [12]: TRL 1 - Princípios básicos observados/ reportados; TRL 2 Formulação de conceitos tecnológicos e/ou aplicação; TRL 3 - Função crítica de forma analítica ou experimental e ou prova de conceito; TRL 4 - Validação funcional dos componentes em ambiente de laboratório; TRL 5 - Validação das funções críticas dos componentes em ambiente relevante; TRL 6 - Demonstração de funções críticas do protótipo em ambiente relevante; TRL 7 - Demonstração de protótipo do Sistema e ambiente operacional; TRL 8 - Sistema qualificado e finalizado; TRL 9 - Sistema operando em todos os aspectos de sua missão operacional.

\section{METODOLOGIA}

A metodologia adotada para esse estudo foi a revisão integrativa da literatura, cuja etapas são: $1^{\circ}$ Fase- Identificação das Perguntas de Pesquisa; $2^{\circ}$ Fase- Critérios de Inclusão e Exclusão; 3 Fase- Coleta de Dados e Filtro dos Artigos; 4ํFase- Análise crítica através da Qualificação; 5 Fase- Discussão dos Resultados; 6ำ FaseApresentação da revisão integrativa.

\section{1. $1^{\circ}$ Fase: Identificação das Perguntas de Pesquisa}

Foram definidas as questões norteadoras para início das buscas: P1- Existe gerenciamento de riscos no processo de desenvolvimento de tecnologia, e novos produtos? P2- A maturidade da tecnologia é considerada? Logo, as buscas foram realizadas nos repositórios do Google Acadêmico, Science Direct, WEB of Science e Scopus no mês de julho 2019. Segue o conjunto de descritores e combinações utilizados: "Gerenciamento de Riscos", "Desenvolvimento de Produto e Tecnologia", "Maturidade da Tecnologia"; "Risk Development", "Technology Readiness Level", "Technology Development".

\subsection{Fase: Critérios de Inclusão e Exclusão}

Os critérios para inclusão dos estudos, foram: artigos Inglês ou Português em periódicos, entre os anos 2014 e 2019. Não foram consideradas publicações sem enfoque em projetos complexos, resenhas, dissertações ou teses.

\subsection{Fase- Coleta de Dados e Filtro dos Artigos}

A tabela 1 apresenta os resultados encontrados por etapas, no mês de julho 2019. Foram encontrados 307 artigos. 


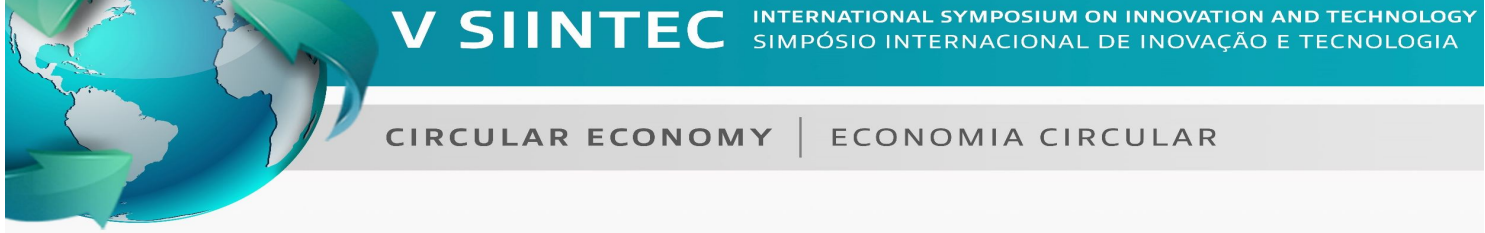

Tabela 1. Total de artigos encontrados

\begin{tabular}{|l|c|c|c|}
\hline \multicolumn{1}{|c|}{ BASE } & ETAPA 1 & ETAPA 2 & ETAPA 3 \\
\hline Google Acadêmico & 106 & 25 & 14 \\
\hline Science Direct & 155 & 17 & 9 \\
\hline Web of Science & 38 & 7 & 2 \\
\hline Scopus & 8 & 1 & 1 \\
\hline
\end{tabular}

A etapa 1 consiste na aplicação dos critérios de inclusão e exclusão, conforme 2.2. Na etapa 2 , buscou-se os termos de identificação no título e resumo, resultando 50 artigos. Na etapa 3, foram considerados apenas artigos cuja metodologia e análise estavam relacionados ao tema proposto, culminando em 26 artigos.

\subsection{Fase- Análise Crítica Através da Qualificação}

Assim, os artigos resultantes da etapa 3 foram avaliados através das perguntas qualificadoras: P1- E considerada a análise de TRL em conjunto com a análise de riscos? (Peso 2); P2- É considerada alguma ferramenta para gerenciamento do risco (qualitativo ou quantitativo)? (Peso 1); P3- É abordado o desenvolvimento de novos produtos complexos e multidisciplinares? (Peso 1); P4- É considerado a avaliação de outras categorias como fonte de risco (Meio Ambiente e Segurança), (Peso 1)? Assim, cada pergunta foi respondida com 1 (Atende), 0,5 (Atende Parcialmente) e 0 (Não atende). Logo, os trabalhos com nota maior ou igual a 4, cuja nota de P1 for igual a 1 , foram selecionados como relevantes.

Tabela 2. Qualificação dos artigos selecionados pela etapa 3.

\begin{tabular}{|c|c|c|c|c|c|c|c|}
\hline № & Titulo & Ano & P1 & P2 & P3 & P4 & Total \\
\hline 1 & A Product-Based Strategic Technology Management.. & 2019 & 0,5 & 1 & 0,5 & 0 & 2,5 \\
\hline 2 & Integrating Product and Technology Development & 2019 & 0,5 & 1 & 1 & 0 & 3 \\
\hline 3 & Applying the Smart Grid Architecture Model.. & 2019 & 1 & 0,5 & 1 & 0,5 & 4 \\
\hline 4 & Applying system readiness levels to cost estimates & 2018 & 1 & 1 & 0,5 & 0,5 & 4 \\
\hline 5 & Use of TRL in the systems engineering toolbox & 2018 & 1 & 1 & 0,5 & 0,5 & 4 \\
\hline 6 & Assessment of active methods.. & 2018 & 0,5 & 0 & 0 & 0 & 1 \\
\hline 7 & Incorporating Human Readiness Levels & 2018 & 1 & 0 & 1 & 0 & 3 \\
\hline 8 & Readiness, feasibility and confidence & 2018 & 1 & 0,5 & 1 & 1 & 4,5 \\
\hline 9 & Operations in Space: Exploring a New Industry & 2018 & 0 & 0 & 0,5 & 0 & 0,5 \\
\hline 10 & System Readiness Level Estimation of Oil and Gas & 2018 & 1 & 0,5 & 1 & 0 & 3,5 \\
\hline 11 & Sustainability of Additive Manufacturing & 2018 & 0,5 & 0,5 & 1 & 0 & 2,5 \\
\hline 12 & Technology Readiness Assessment & 2018 & 1 & 0 & 1 & 0 & 3 \\
\hline 13 & Using TRLS and System Architecture & 2017 & 1 & 1 & 1 & 0 & 4 \\
\hline 14 & Three dimensions of maturity... & 2017 & 0,5 & 1 & 0 & 1 & 3 \\
\hline 15 & A Survey of Technology Readiness Level Users & 2016 & 1 & 1 & 1 & 0 & 4 \\
\hline 16 & Methodology for Risk Assessment & 2016 & 1 & 1 & 1 & 0 & 4 \\
\hline 17 & A Survey of Technology Readiness Level Users & 2016 & 0,5 & 0 & 0 & 0 & 1 \\
\hline 18 & Risk vs. Reward: A Methodology & 2016 & 0 & 1 & 1 & 0 & 2 \\
\hline 19 & The interdiscursive appeal of risk matrices & 2016 & 0,5 & 1 & 1 & 1 & 4 \\
\hline 20 & Technology risk assessment .. & 2016 & 1 & 0,5 & 1 & 1 & 4,5 \\
\hline 21 & Technology planning approach for Very Small Entities & 2016 & 0 & 1 & 0,5 & 0 & 1,5 \\
\hline 22 & In search of technology readiness level (TRL)10 & 2015 & 1 & 0 & 1 & 0 & 3 \\
\hline 23 & On the use of directional and incremental prototyping & 2015 & 0 & 0 & 1 & 0 & 1 \\
\hline 24 & A combined technique using SEM and TOPSIS & 2015 & 0,5 & 0,5 & 1 & 0 & 2,5 \\
\hline 25 & Incidente Matrix Approach for Calculating Readiness.. & 2014 & 1 & 1 & 0 & 0 & 3 \\
\hline 26 & A Performance-Based System Maturity Assessment & 2014 & 0,5 & 1 & 0,5 & 0 & 2,5 \\
\hline
\end{tabular}




\section{SIINTEC INTERNATIONAL SYMPOSIUM ON INNOVATION AND TECHNOLOGY}

CIRCULAR ECONOMY

ECONOMIA CIRCULAR

Tabela 3. Validação da qualidade

\begin{tabular}{|l|l|l|l|l|l|l|l|}
\hline \multicolumn{2}{|c|}{ Titulo } & Ano & P1 & P2 & P3 & P4 & Nota \\
\hline 1 & Integrating Product and Technology Development & 2019 & 1 & 0,5 & 1 & 0,5 & 4 \\
\hline 2 & Applying the Smart Grid Architecture Model for Designing & 2019 & 1 & 0,5 & 1 & 0,5 & 4 \\
\hline 3 & Applying system readiness levels to cost estimates & 2018 & 1 & 1 & 0,5 & 0,5 & 4 \\
\hline 4 & Readiness, feasibility and confidence & 2018 & 1 & 0,5 & 1 & 1 & 4,5 \\
\hline 5 & System Readiness Level Estimation of Oil and Gas Production & 2018 & 1 & 0,5 & 1 & 0,5 & 4 \\
\hline 6 & Using TRLS and System Architecture & 2017 & 1 & 1 & 1 & 0 & 4 \\
\hline 7 & A Survey of Technology Readiness Level Users & 2016 & 1 & 1 & 1 & 0 & 4 \\
\hline 8 & Methodology for Risk Assessment of Substructures & 2016 & 1 & 1 & 1 & 0 & 4 \\
\hline 9 & Technology risk assessment as part of risk assessment & 2016 & 1 & 0,5 & 1 & 1 & 4,5 \\
\hline 10 & Incidence Matrix Approach For Calculating Readiness Levels & 2014 & 1 & 1 & 0,5 & 0,5 & 4 \\
\hline
\end{tabular}

Baseado no processo de qualificação, dos 26 artigos avaliados, restaram 10 estudos com notas iguais ou maiores que 4,0 .

\subsection{5ํFase- Discussão dos Resultados}

Baseado na análise dos artigos selecionados, foi observado que o TRL sozinho não garante a avaliação e tratamento dos riscos do projeto. Ou seja, métodos complementares devem ser implementados para identificar incertezas e ameaças durante o processo de desenvolvimento do produto. Foi ressaltado em um dos artigos, o desafio da interpretação das métricas do TRL e sua aplicabilidade com projetos de natureza complexa [13], porém, o artigo não menciona como melhorar o entendimento do TRL para validar o projeto.

Observou-se também, que, embora os TRLs fossem avaliados principalmente ao nível do componente do produto, a equipe de engenharia deve estar atenta se a integração da tecnologia está sendo considerada. [14,19,17]. Mesmo mencionando a importância da integração da tecnologia, os trabalhos em questão não mencionam como analisar possíveis riscos no processo de validação da TRL, considerando a severidade e a probabilidade do risco.

A métrica IRL (Integration Readiness Leve/) auxilia no processo de integração das tecnologias de acordo com a quantidade de interfaces propostas por cada componente [20]. A aplicação da IRL nas fases do TRL possibilita o amadurecimento do sistema [15, 22]. Porém, ainda se percebe a falta de métricas em como as ameaças e incertezas provenientes da integração da tecnologia devem ser reconhecidos e tratados.

Em um dos trabalhos, foi considerado o método que avalia a probabilidade de propagação do risco e seu impacto no sistema. Esse método não envolve a TRL diretamente. Em vez disso, exige que os profissionais do projeto realizem avaliações em cada componente do desenvolvimento do produto que compartilhem a mesma interface ou arquitetura [16]. Ao fim, espera-se que apenas a experiência dos 


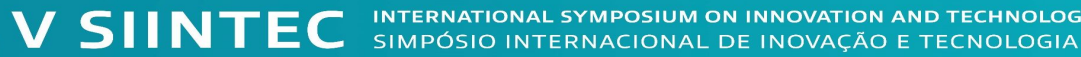

engenheiros consiga identificar os riscos de cada processo do desenvolvimento sem a avaliação da maturidade da tecnologia e respectivos riscos.

Outro estudo propõe avaliar a prontidão do sistema para a tomada de decisão e então quantificar o nível de integração de cada componente melhorando o produto, tal como o processo de fabricação e instalação, [21] mas não é mencionado relação direta em como os riscos do projeto no processo de integração.

Foi verificada uma proposta [16] que se concentrou nos conceitos de mudança de engenharia e a propagação dessas mudanças. Tratando-se de sistemas altamente complexos, a mudança na engenharia é necessária para solucionar possíveis riscos e incertezas durante o projeto. Visto isso, o trabalho afirma que o risco de integração da tecnologia de cada componente deve ser estimado utilizando métodos temporais, como a teoria de Grafos [17]. Com isso, a análise da estrutura do sistema forneceria não é confiável devido a limitação que uma matriz ordinal representa.

A aplicação de uma escala SRL (system readiness level), possibilita identificar o nível de maturidade em todo o sistema ( $I R L+T R L)$, para projetos complexos que envolvam incertezas [21]. Porém, a lacuna encontrada nesse processo é que os fatores negativos ou ameaças estão limitadas apenas ao custo ou a qualidade do projeto, não sendo considerados outros fatores do projeto, como meio ambiente e segurança.

Outro trabalho menciona uma métrica adotada chamada $A R L$ (Activity Risk Level), utilizando uma escala de probabilidade e impacto [20], mas considerando apenas riscos de natureza técnica, sem vincular o TRL em cada etapa do desenvolvimento.

O uso da TRL se expandiu rapidamente da NASA para o departamento de Defesa dos Estado Unidos. Nesse processo, foram identificadas limitações da métrica sendo necessário algumas adaptações: Na fase 1 e 2 da TRL é agregado o método Material Solution Analysis (MSA), na fase 3, 4 e 5 da TRL equivale ao processo de Technology Demonstration (TD), as fases 6 e 7 da TRL utiliza o Development (EMD). Já nas fases finais da TRL 8 e 9, o departamento de defesa $(D o D)$ acrescenta o Operations \& Support. Esse processo de agregação de métricas permite a contemplação dos requisitos [17]. Por outro lado, essa agregação de métricas não tem por objetivo a análise impactos negativos e probabilidade de riscos no contexto tecnológico, além de não mencionar os fatores ambientais como possíveis fatores de riscos.

\subsection{Fase- Apresentação da revisão integrativa}

Na presente revisão integrativa foram encontrados 2 artigos de 2019, 3 artigos de 2018, 1 artigo de 2017, 3 artigos de 2016 e 1 artigo de 2014. Nesse resultado, percebe-se que $100 \%$ dos artigos são internacionais.

Percebe-se que ainda existem lacunas referente aos trabalhos selecionados, pois, os mesmos não consideram o uso do TRL associados a uma ferramenta para levantamento e tratamento do risco (seja qualitativa ou quantitativa). Os resultados ainda apontam a falta de dados sobre avaliação das categorias como Meio Ambiente 


\section{SIINTEC INTERNATIONAL SYMPOSIUM ON INNOVATION AND TECHNOLOG}

e Segurança. Essas categorias devem ser consideradas, pois, todos os impactos ou ameaças que envolvem o ambiente do projeto devem ser conhecidos, avaliados e tratados. Outro fator de fundamental importância nesse processo, é a necessidade de considerar a etapa do desenvolvimento para levantamento dos riscos em uma frequência estabelecida, sempre utilizando o nível de criticidade do risco como parâmetro para a priorização das ações.

\section{CONCLUSÃO}

Observou-se com esse estudo, que os métodos aplicados para gerenciamento de riscos no desenvolvimento de tecnologias e novos produtos é diversificado devido à natureza dos projetos. Na maior parte dos trabalhos, verificou-se que são considerados o uso do TRL agregado a outra ferramenta para verificação da qualificação da tecnologia, porém, mesmo agregando ferramentas para análise da maturidade tecnológica, não foram considerados métodos para identificação dos riscos agregados, tanto para riscos técnicos e gerenciais, quanto para o meio ambiente e segurança.

Foi observado que poucos autores consideraram a avaliação da TRL por fase do desenvolvimento do produto, já que as fases, assim como os níveis da TRL, possuem características especificas para o avanço do desenvolvimento e confirmação da validação da tecnologia proposta. Observou-se ainda a falta de abordagem sobre os riscos inerentes a multidisciplinaridade dos projetos de tecnologia.

Com isso, esse estudo evidencia uma lacuna sobre trabalhos que referencie métodos de gerenciamento de ameaças, considerando os fatores ambientais, de segurança, gerenciais e técnicos, tendo como base as métricas do TRL, em cada etapa do desenvolvimento.

A próxima etapa para esse trabalho, consiste em desenvolvimento de uma ferramenta de gestão de riscos que possibilite análise das ameaças e impactos negativos em cada etapa do desenvolvimento, considerando o nível de maturidade tecnológica e respectiva integração dos componentes e tecnologias envolvidas, tal como a análise do meio ambiente e segurança, a fim de evitar retrabalhos e não conformidades do produto e do projeto.

\section{Agradecimentos}

Agradeço ao SENAI CIMATEC por promover a bolsa de estudos (ANP), para viabilizar essa pesquisa, cujo objetivo é integrar a Gestão de Riscos na metodologia de desenvolvimento de produtos e tecnologia.

\section{REFERÊNCIAS}

${ }^{1}$ Grubisic, V., 2009. Metodologia de gerenciamento integrado de riscos técnicos e gerenciais para o projeto de produtos. Universidade Federal De Santa Catarina, Programa De Pós-Graduação Em Engenharia Mecânica, Florianópolis. 
2 Olechowski A., Oehmen, J., Seering, W., Ben-Daya, M., 2016. The professionalization of risk management: What role can the ISO 31000 risk management principles play? International Journal of Project Management, 1568-1578.

${ }^{3}$ REZENDE, Leandro Bolzan; BLACKWELL, Paul. Revisiting project complexity: a new dimension and framework. The Journal of Modern Project Management, v. 6, n. 3, 2019.

${ }^{4}$ Shah, L. et al. 2016. Decision-making in the manufacturing using a value-risk graph. Journal of Intelligent Manufacturing, p. 617-630, 2016.

${ }^{5}$ ISO 31000:2009. Risk management - Principles and guidelines. ABNT, RJ.

6 Willumsen, P., Oehmen, J., Stingl, V., Geraldi, J., Value creation through project risk management, International Journal of Project Management, 2019.

7 Matsumoto, S. M. 2010. Proposta de Método para a Gestão de Riscos em Projetos de Inovação Tecnológica. Instituo Tecnológico de Aeronáutica, Campo Montenegro, São José dos Campos, SP, Brasil.

${ }^{8}$ Talbot, J., 2008. What's right with risk matrices? Juliantalbot.com. Risk Management Policy.

${ }^{9}$ ISO14040, 2006. Environmental Management: Life Cycle Assessment: Principles and Framework. Technical Committee ISO/TC 207.

10API 17Q, Recommended Practice on Subsea Equipment Qualification, American Petroleum Institute, Second Edition, May 2018.

11 Yang, K., Zhao, R., \& lan, Y., 2014. The impact of risk attitude in new product development under dual information asymmetry. Computers \& Industrial Engineering, 76, 122-137.

12 Makins, J. C., 2004. Technology Readiness Levels. A White Paper. Advanced Concepts Office. Office of Space Access and Technology NASA.

13 Tomaschek, K., Olechowski, A., Joglekar, N, Eppinger, S. A Survey of Technology Readiness Level Users. INCOSE International Symposium, July 18-21, 2016.

${ }^{14}$ Malone, P.,K,. Applying system readiness levels to cost estimates - A case study. IEEE, Continental Suite 185 El Segundo, CA 90245 310-640-0005. 2018.

${ }^{15}$ Garg, T., Eppinger, S.,Joglekar, N., Olechowski, A., Using TRLS and System Architecture to Estimate Technology Integration Risk. Massachusetts Institute of Technology, International Conference On Engineering Design, Iced17, 2017.

${ }^{16}$ London, M., A., Holzer, H. T., Eveleigh, J., T., Sarkani, S. Incidence Matrix Approach For Calculating Readiness Levels. School of Engineering and Applied Science, 1861-9576, 2016.

${ }_{17}$ Proskovics, R., Hutton, G., Torr, R., Scheu, M.N., Methodology for Risk Assessment of Substructures for Floating Wind Turbines. Energy Procedia 45-82, 2016.

${ }^{18}$ Brilhuis-Meijer. E, Pigosso, D., C., A., McAloone, T., M. Integrating product and technology development: A proposed reference model for dual innovation. 26th CIRP Design Conference $32-37.2016$.

19 Sylla, A., Vareilles, E., Coudert, T., Konstantinos K., Aldanondo M., Geneste, L., Readiness, feasibility and confidence: how to help bidders to better development. International Journal of Production Research, vol. 55 ( $n^{\circ}$ 23). pp. 7204-7222. 2017.

20 Yasseri, S. F., Bahai, H. System Readiness Level Estimation of Oil and Gas Production Systems. International Journal Of Coastal \& Offshore Engineering, 31-44. 2018.

${ }^{21}$ Toljaga-Nikolić, D., Technology risk assessment as part of risk assessment as part of risk management process.Serbian Project Management Association, Vol 6, 2016.

22 Uslar, M., Rohjans, S., Neureiter, S., Andrén, F., P., Velasquez, J., Steinbrink, J., Efthymiou, V., Migliavacca, G., Horsmanheimo, S., Brunner, S., Thomas, S., Strasserl., Applying the Smart Grid Architecture Model for Designing and Validating System-of-Systems in the Power and Energy Domain: A European Perspective. Energies, 2019 